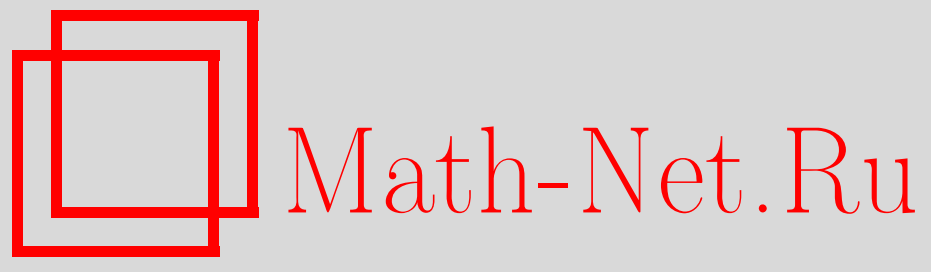

В. А. Кондратьев, И. А. Рудаков, О периодических решениях квазилинейного волнового уравнения, Матем. заметки, 2009, том 85, выпуск 1, 36-53

DOI: https://doi.org/10.4213/mzm6584

Использование Общероссийского математического портала Math-Net.Ru подразумевает, что вы прочитали и согласны с пользовательским соглашением http://www . mathnet.ru/rus/agreement

Параметры загрузки:

IP : 54.197 .217 .227

26 апреля 2023 г., 16:19:18

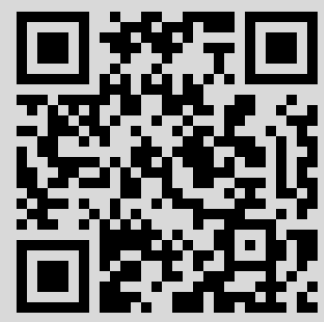


Том 85 выпуск 1 январь 2009

\author{
УДК 517.956.35
}

\title{
О периодических решениях квазилинейного волнового уравнения
}

\section{В. А. Кондратьев, И. А. Рудаков}

Исследуются свойства волнового оператора с условием периодичности по времени и однородными граничными условиями 3-го рода и Дирихле. Доказано существование нетривиального периодического по времени решения sin-Гордон с однородными граничными условиями 3-го рода и Дирихле. Получены теоремы о существовании периодического решения квазилинейного волнового уравнения с переменными по $x$ коэффициентами и граничным условием 3 -рода.

Библиография: 16 названий.

Введение. Рассматривается задача

$$
\begin{array}{ccc}
u_{t t}-u_{x x}=g(u)+f(x, t), & 0<x<\pi, & t \in \mathbb{R}, \\
u(x, t+T)=u(x, t), & 0<x<\pi, \quad t \in \mathbb{R} .
\end{array}
$$

Будем исследовать следующие два типа граничных условий:

$$
\begin{array}{ll}
u(0, t)=u(\pi, t)+h u_{x}(\pi, t)=0, & t \in \mathbb{R}, \\
u(0, t)-h u_{x}(0, t)=u(\pi, t)=0, & t \in \mathbb{R} .
\end{array}
$$

Здесь $T=2 \pi b / a$, где $a, b$ есть взаимно простые натуральные числа и $f(x, t)$ есть заданная $T$-периодическая по времени функция. Задача $(0.1),(0.2)$ с однородными граничными условиями Дирихле изучена в работах [1]-[5]. В работах [6]-[9] доказано существование обобщенных решений задачи (0.1), (0.2) с однородными граничными условиями 3-го рода с константой $h>0$. В работах [10], [11] рассмотрено волновое уравнение с переменными коэффициентами и граничными условиями 3-го рода с $h>0$. Целью данной работы является исследование случая $h<0$ и доказательство теорем о гладкости обобщенных решений задач $(0.1)-(0.3)$ и $(0.1),(0.2),(0.4)$, если функции $g, f$ являются достаточно гладкими.

1. Линейное неоднородное уравнение. Решение задач $(0.1)-(0.3)$ и $(0.1),(0.2)$, (0.4) будем искать в виде ряда Фурье по системе

$$
\left\{\frac{1}{\sqrt{T}} \varphi_{n}(x), \frac{2}{\sqrt{T}} \varphi_{n}(x) \cos \frac{a}{b} m t, \frac{2}{\sqrt{T}} \varphi_{n}(x) \sin \frac{a}{b} m t\right\} .
$$

(C) В.А. Кондратьев, И. А. Рудаков, 2009 
Здесь $\varphi_{n}(x)$ есть нормированные $\left(\left\|\varphi_{n}\right\|_{L_{2}(0, \pi)}=1\right)$ собственные функции соответствующей задачи Штурма-Лиувилля:

$$
\begin{array}{rlrl}
\varphi^{\prime \prime}+\lambda^{2} \varphi & =0, & \varphi(0)=\varphi(\pi)+h \varphi^{\prime}(\pi)=0, \\
\varphi^{\prime \prime}+\lambda^{2} \varphi=0, & \varphi(0)-h \varphi^{\prime}(0)=\varphi(\pi)=0 .
\end{array}
$$

Обозначим $\Omega=[0, \pi] \times \mathbb{R} /(T \mathbb{Z})$ и $\|\cdot\|,(\cdot, \cdot)$ - норму и скалярное произведение в $L_{2}(\Omega)$ соответственно. Система $(1.1)$ является полной ортонормированной системой в $L_{2}(\Omega)$. Легко видеть, что задачи (1.2), (1.3) имеют одинаковые собственные числа $\lambda_{n}$, удовлетворяющие уравнению $\operatorname{tg}\left(\lambda_{n} \pi\right)=-h \lambda_{n}$. Обозначим $\theta_{n}=\lambda_{n}-$ $(n-1 / 2)$. Тогда

$$
\lambda_{n}=n-\frac{1}{2}+\theta_{n}
$$

Не трудно показать, что $\theta_{n} \in(0,1 / 2)$. В [9] показано, что существуют положительные константы $b_{0}, b_{1}$ (в качестве $b_{0}$ можно взять $\left.2 /(h \pi)\right)$ такие, что

$$
0<b_{0} \frac{1}{n}<\theta_{n}<b_{1} \frac{1}{n} \quad \text { для всех } n \in \mathbb{N} .
$$

Простые вычисления показывают, что для задачи (1.2) $\varphi_{n}=C_{n} \sin \lambda_{n} x$, а для задачи (1.3) $\varphi_{n}=C_{n}\left(\sin \lambda_{n} x+\lambda_{n} h \cos \lambda_{n} x\right)$, где $C_{n}=\sqrt{2 / \pi}+\alpha_{n}, \alpha_{n}>0$ и $\lim _{n \rightarrow \infty} \alpha_{n}=0$.

Определим оператор $A_{0}: L_{2}(\Omega) \rightarrow L_{2}(\Omega)$, для которого

$$
D\left(A_{0}\right)=\left\{u=\sum_{n=1}^{N} \sum_{m=1}^{M} \varphi_{n}\left(a_{n m} \cos \frac{a}{b} m t+b_{n m} \sin \frac{a}{b} m t\right) \mid M, N \in \mathbb{N}\right\}
$$

и $A_{0} \varphi=\varphi_{t t}-\varphi_{x x}$ для любого $\varphi \in D\left(A_{0}\right)$. Пусть $A=A^{*}$ в $L_{2}(\Omega)$. Система функций (1.1) является системой собственных функций операторов $A_{0}$ и $A$ с собственными значениями

$$
\mu_{n m}=\lambda_{n}^{2}-\left(\frac{a}{b} m\right)^{2}=\left(n-\frac{1}{2}+\theta_{n}\right)^{2}-\left(\frac{a}{b} m\right)^{2}
$$

Оператор $A$ самосопряжен в $L_{2}(\Omega)$, образ $R(A)$ замкнут в $L_{2}(\Omega)$,

$$
L_{2}(\Omega)=R(A) \oplus \operatorname{ker} A, \quad \operatorname{dim} \operatorname{ker} A<\infty .
$$

При $a=1$ (см. [7]) $\operatorname{ker} A=\{0\}$. Рассмотрим линейное неоднородное уравнение

$$
u_{t t}-u_{x x}=f(x, t), \quad 0<x<\pi, \quad t \in \mathbb{R} .
$$

Здесь $f$ есть заданная $T$-периодическая функция из $L_{2}(\Omega)$.

ОПРЕДЕЛЕНИЕ. Обобщенным решением задач $(1.6),(0.2),(0.3)$ и $(1.6),(0.2),(0.4)$ называется $T$-периодическая по $t$ функция $u \in L_{2}(\Omega)$ такая, что

$$
\left(u, \varphi_{t t}-\varphi_{x x}\right)=(f, \varphi) \quad \text { для всех } \varphi \in D\left(A_{0}\right) .
$$

Обозначим $H_{k}(\Omega)=W_{k}^{2}(\Omega)$ пространство Соболева и $\|\cdot\|_{k}=\|\cdot\|_{H_{k}(\Omega)}, H_{0}(\Omega)=$ $L_{2}(\Omega)$. $T$-периодическая по $t$ функция $u$ является обобщенным решением задач (1.6), $(0.2),(0.3)$ и $(1.6),(0.2),(0.4)$ тогда и только тогда, когда $u \in D(A)$ и $A u=f$. 
Tеорема 1. Пусть $h>0$, b есть нечетное число $u k \in\{0,1\}$. Тогда для любой $T$-периодической по $t$ функции $f \in H_{k}(\Omega) \cap R(A)$, задачи (1.6),(0.2),(0.3) u (1.6), $(0.2),(0.4)$ имеют обобщенное решение $u \in H_{k+1}(\Omega)$. Это решение единственно в подпространстве $H_{k+1}(\Omega) \cap R(A)$ и существует положительная константа $C_{k}$, не зависимая от $f$, такая, что

$$
\left\|A^{-1} f\right\|_{k+1} \leqslant C_{k}\|f\|_{k} \quad \text { для всех } f \in H_{k}(\Omega) \cap R(A) .
$$

Eсли $f \in H_{1}(\Omega) \cap R(A)$, mo $A^{-1} f \in H_{2}(\Omega) \cap C^{1}(\Omega)$.

ДоказАтеЛЬСтво. Разложим функцию $f \in H_{k}(\Omega) \cap R(A)$ в ряд Фурье по системе (1.1)

$$
f=\sum_{n=1}^{\infty} f_{n} \varphi_{n}(x)+\sum_{\substack{n, m \in \mathbb{N} \\ \mu_{n m} \neq 0}} \varphi_{n}(x)\left(a_{n m} \cos \frac{a}{b} m t+b_{n m} \sin \frac{a}{b} m t\right) .
$$

Тогда

$$
u=A^{-1} f=\sum_{n=1}^{\infty} \frac{1}{\lambda_{n}^{2}} f_{n} \varphi_{n}(x)+\sum_{\substack{n, m \in \mathbb{N} \\ \mu_{n m} \neq 0}} \frac{1}{\mu_{n m}} \varphi_{n}(x)\left(a_{n m} \cos \frac{a}{b} m t+b_{n m} \sin \frac{a}{b} m t\right) .
$$

Обозначим $u_{1}=\sum_{n=1}^{\infty}\left(1 / \lambda_{n}^{2}\right) f_{n} \varphi_{n}(x), u_{2}=u-u_{1}$. Легко видеть, что

$$
\left|\varphi_{n}^{\prime}(x)\right| \leqslant C \lambda_{n} \quad \text { для всех } \quad x, n,
$$

где $C$ не зависит от $x$ и $n$. Поэтому

$$
\sum_{n=1}^{\infty} \frac{1}{\lambda_{n}^{2}}\left|f_{n} \varphi_{n}^{\prime}(x)\right| \leqslant C\left(\sum_{n=1}^{\infty} \frac{1}{\lambda_{n}^{2}} \sum_{n=1}^{\infty} f_{n}^{2}\right)^{1 / 2}<\infty
$$

и $u \in C^{1}(0, \pi)$. Поскольку $\varphi_{n}^{\prime \prime}(x)=-\lambda_{n}^{2} \varphi_{n}(x)$, то

$$
u_{1} \in H_{2}(0, \pi) \cap C^{1}(0, \pi) .
$$

Рассмотрим ряд

$$
\sum_{\substack{n, m \in \mathbb{N} \\ \mu_{n m} \neq 0}} \frac{m}{\mu_{n m}} \varphi_{n}(x)\left(-a_{n m} \sin \frac{a}{b} m t+b_{n m} \cos \frac{a}{b} m t\right) .
$$

Учитывая нечетность $b$, оценим множитель

$$
\begin{aligned}
\left|\frac{m}{\mu_{n m}}\right| & =\frac{m}{\left|n-\frac{1}{2}+\theta_{n}-\frac{a}{b} m\right|\left(n-\frac{1}{2}+\theta_{n}+\frac{a}{b} m\right)}<\frac{b^{2}}{a} \frac{1}{\left|b n-a m-\frac{b}{2}+b \theta_{n}\right|} \\
& \leqslant \frac{b^{2}}{a|| b n-a m-\frac{b}{2}\left|-b \theta_{n}\right|} \leqslant \frac{b^{2}}{a\left|\frac{1}{2}-b \theta_{n}\right|} \leqslant C_{1},
\end{aligned}
$$


если $\mu_{n m} \neq 0$ и $\theta_{n} \in(0,1 /(2 b))$. А поскольку $\theta_{n} \rightarrow 0$, ряд $(1.9)$ сходится в $L_{2}(\Omega)$ и $\left(u_{2}\right)_{t} \in L_{2}(\Omega)$.

Рассмотрим вначале граничные условия (0.3) и напишем ряд

$$
\begin{aligned}
\sum_{\substack{n, m \in \mathbb{N} \\
\mu_{n m} \neq 0}} \frac{1}{\mu_{n m}} \varphi_{n}^{\prime}(x)\left(a_{n m} \cos \frac{a}{b} m t+b_{n m} \sin \frac{a}{b} m t\right) \\
=\sum_{\substack{n, m \in \mathbb{N} \\
\mu_{n m} \neq 0}} C_{n} \frac{\lambda_{n}}{\mu_{n m}} \cos \lambda_{n} x\left(a_{n m} \cos \frac{a}{b} m t+b_{n m} \sin \frac{a}{b} m t\right) .
\end{aligned}
$$

Система функций $\left\{\cos \lambda_{n} x\right\}$ не является ортогональной в $L_{2}(0, \pi)$. Чтобы исследовать сходимость ряда (1.11), разложим функцию $\cos \lambda_{n} x$ в ряд Фурье по системе $\varphi_{k}(x)=C_{k} \sin \lambda_{k} x:$

$$
\cos \lambda_{n} x=\sum_{k=1}^{\infty} \gamma_{k n} \cdot \varphi_{k}(x)
$$

где

$$
\gamma_{k k}=C_{k} \int_{0}^{\pi} \cos \lambda_{k} x \sin \lambda_{k} x d x=\frac{1}{4 \lambda_{k}} C_{k}\left(1-\cos 2 \lambda_{k} \pi\right)=\frac{1}{2} C_{k} \cdot \frac{\sin ^{2}\left(\lambda_{k} \pi\right)}{\lambda_{k}}
$$

и при $k \neq n$

$$
\gamma_{k n}=\frac{1}{2} C_{k}\left(\frac{1}{\lambda_{k}-\lambda_{n}}+\frac{1}{\lambda_{k}+\lambda_{n}}-\frac{\cos \left(\lambda_{k}-\lambda_{n}\right) \pi}{\lambda_{k}-\lambda_{n}}-\frac{\cos \left(\lambda_{k}+\lambda_{n}\right) \pi}{\lambda_{k}+\lambda_{n}}\right) .
$$

Следовательно, существует константа $C_{2}>0$ такая, что

$$
\left|\gamma_{k n}\right| \leqslant \frac{C_{2}}{\left|\lambda_{k}-\lambda_{n}\right|}, \quad\left|\gamma_{k k}\right| \leqslant \frac{C_{2}}{\lambda_{k}}
$$

Оценим сумму ряда

$$
\begin{aligned}
I & =\sum_{k=1}^{\infty} \sum_{m=1}^{\infty}\left(\sum_{\substack{n \in \mathbb{N} \\
\mu_{n m} \neq 0}}\left|\frac{\lambda_{n} \gamma_{k n} a_{n m}}{\mu_{n m}}\right|\right)^{2} \\
& \leqslant \sum_{k=1}^{\infty} \sum_{m=1}^{\infty}\left(\sum_{n=1}^{\infty} a_{n m}^{2}\right)\left(\sum_{\substack{l \in \mathbb{N} \\
\mu_{l m} \neq 0}} \frac{\lambda_{l}^{2} \gamma_{k l}^{2}}{\mu_{l m}^{2}}\right)=\sum_{m=1}^{\infty} \sum_{n=1}^{\infty} a_{n m}^{2} \sum_{k=1}^{\infty} \sum_{\substack{l \in \mathbb{N} \\
\mu_{l m} \neq 0}} \frac{\lambda_{l}^{2} \gamma_{k l}^{2}}{\mu_{l m}^{2}}
\end{aligned}
$$

Пусть

$$
S_{m}=\sum_{k=1}^{\infty} \sum_{\substack{l \in \mathbb{N} \\ \mu_{l m} \neq 0}} \frac{\lambda_{l}^{2} \gamma_{k l}^{2}}{\mu_{l m}^{2}}=\sum_{\substack{k \in \mathbb{N} \\ \mu_{k m} \neq 0}} \frac{\lambda_{k}^{2} \gamma_{k k}^{2}}{\mu_{k m}^{2}}+\sum_{k=1}^{\infty} \sum_{\substack{l \neq k \\ \mu_{l m} \neq 0}} \frac{\lambda_{l}^{2} \gamma_{k l}^{2}}{\mu_{l m}^{2}}
$$

Легко видеть, что существует константа $C_{0}>0$ такая, что если $\mu_{k m} \neq 0$, то $\left|\mu_{k m}\right| \geqslant$ $C_{0}|k+(a / b) m-1 / 2|$. Отсюда и из (1.13) выведем

$$
\sum_{\substack{k \in \mathbb{N} \\ \mu_{k m} \neq 0}} \frac{\lambda_{k}^{2} \gamma_{k k}^{2}}{\mu_{k m}^{2}} \leqslant \frac{C_{2}^{2}}{C_{0}^{2}} \sum_{k=1}^{\infty} \frac{1}{(k-1 / 2)^{2}}=\frac{\pi^{2} C_{2}^{2}}{2 C_{0}^{2}} .
$$


Используя (1.13), оценим второе слагаемое:

$$
\begin{aligned}
\sum_{k=1}^{\infty} \sum_{\substack{l \neq k \\
\mu_{l m} \neq 0}} \frac{\lambda_{l}^{2} \gamma_{k l}^{2}}{\mu_{l m}^{2}} & =\sum_{\substack{l=1 \\
\mu_{l m} \neq 0}}^{\infty} \frac{\lambda_{l}^{2}}{\mu_{l m}^{2}} \sum_{\substack{k=1 \\
k \neq l}}^{\infty} \gamma_{k l}^{2} \\
& \leqslant C_{2}^{2} \sum_{\substack{l=1 \\
\mu_{l m} \neq 0}}^{\infty} \frac{\lambda_{l}^{2}}{\left(l-\frac{1}{2}+\theta_{l}-\frac{a}{b} m\right)^{2}\left(\lambda_{l}+\frac{a}{b} m\right)^{2}} \sum_{\substack{k=1 \\
k \neq l}}^{\infty} \frac{1}{\left(\lambda_{k}-\lambda_{l}\right)^{2}} \\
& \leqslant 8 C_{2}^{2} b^{2} \sum_{\substack{l=1 \\
\mu_{l m} \neq 0}}^{\infty} \frac{1}{\left(2 b l-2 a m-b+b \theta_{l}\right)^{2}} \sum_{k=1}^{\infty} \frac{1}{(k-1 / 2)^{2}} .
\end{aligned}
$$

Обозначим через $l_{0}$ такое натуральное число, что $b \theta_{l}<1 / 2$ при любом $l \geqslant l_{0}$. Поскольку $2 b l-2 a m-b$ есть нечетное число, то

$$
\begin{aligned}
\sum_{k=1}^{\infty} \sum_{\substack{l \neq k \\
\mu_{l m} \neq 0}} \frac{\lambda_{l}^{2} \gamma_{k l}^{2}}{\mu_{l m}^{2}} \leqslant & 4 \pi^{2} C_{2}^{2} b^{2} \sum_{\substack{l=1 \\
\mu_{l m} \neq 0}}^{l_{0}-1} \frac{1}{\left(2 b l-2 a m-b+b \theta_{l}\right)^{2}} \\
& +4 \pi^{2} C_{2}^{2} b^{2} \sum_{l=l_{0}}^{\infty} \frac{1}{(|2 b l-2 a m-b|-1 / 2)^{2}} \\
\leqslant & +8 \pi^{2} C_{2}^{2} b^{2} \sum_{k=1}^{\infty} \frac{1}{(k-1 / 2)^{2}}=L_{0}+4 \pi^{4} C_{2}^{2} b^{2}
\end{aligned}
$$

где

$$
L_{0}=4 \pi^{2} C_{2}^{2} b^{2} \sum_{\substack{l=1 \\ \mu_{l m} \neq 0}}^{l_{0}-1} \frac{1}{\min _{m \in \mathbb{N}}\left(2 b l-2 a m-b+b \theta_{l}\right)^{2}} .
$$

Из (1.15), (1.16) и равенства Парсеваля вытекает сходимость ряда I. Аналогично доказывается сходимость ряда

$$
\sum_{k=1}^{\infty} \sum_{m=1}^{\infty}\left(\sum_{\substack{n \in \mathbb{N} \\ \mu_{n m} \neq 0}}\left|\frac{\lambda_{n} \gamma_{k n} b_{n m}}{\mu_{n m}}\right|\right)^{2}
$$

Обозначим

$$
v_{M N}=\sum_{m=1}^{M} \sum_{n=1}^{N} C_{n} \frac{\lambda_{n} a_{n m}}{\mu_{n m}} \cos \lambda_{n} x \cos \frac{a}{b} m t .
$$

Из сходимости ряда $I$ следует существование функции $v \in L_{2}(\Omega)$ такой, что

$$
v=\sum_{k=1}^{\infty} \sum_{m=1}^{\infty}\left(\sum_{\substack{n \in \mathbb{N} \\ \mu_{n m} \neq 0}} C_{n} \frac{\lambda_{n} \gamma_{k n} a_{n m}}{\mu_{n m}}\right) \varphi_{k}(x) \cos \frac{a}{b} m t
$$

Покажем, что последовательность $v_{M N}$ содержит сходящуюся к $v$ в $L_{2}(\Omega)$ подпоследовательность. Обозначим

$$
c_{k m}=\sum_{\substack{n \in \mathbb{N} \\ \mu_{n m} \neq 0}} C_{n} \frac{\sqrt{T} \lambda_{n} \gamma_{k n} a_{n m}}{\sqrt{2} \mu_{n m}}, \quad e_{m}=\sqrt{\frac{2}{T}} \cos \frac{a}{b} m t, \quad e_{k m}=\varphi_{k}(x) e_{m} .
$$


Тогда

$$
v=\sum_{k=1}^{\infty} \sum_{m=1}^{\infty} c_{k m} e_{k m}=\bar{v}_{M K}+\alpha_{M K},
$$

где $\bar{v}_{M K}=\sum_{k=1}^{K} \sum_{m=1}^{M} c_{k m} e_{k m}$. Возьмем произвольное $l \in \mathbb{N}$. Из (1.18) следует существование натуральных чисел $M_{1}=M_{1}(l), K_{1}=K_{1}(l)$ таких, что $M_{1}(l) \rightarrow \infty$, $K_{1}(l) \rightarrow \infty$ при $l \rightarrow \infty$ и при любых $M \geqslant M_{1}, K \geqslant K_{1}$ выполнено неравенство

$$
\left\|\alpha_{M K}\right\|<\frac{1}{3 l} \text {. }
$$

Воспользовавшись введенными выше обозначениями, напишем

$$
\begin{aligned}
v_{M N} & =v+\left(v_{M N}-v\right)=v+\left(v_{M N}-\bar{v}_{M K}\right)-\alpha_{M K}, \\
v_{M N} & =\sum_{m=1}^{M} \sum_{n=1}^{N} C_{n} \frac{\sqrt{T} \lambda_{n} a_{n m}}{\sqrt{2} \mu_{n m}}\left(\sum_{k=1}^{\infty} \gamma_{k n} \varphi_{k}(x)\right) e_{m} \\
& =\sum_{m=1}^{M} \sum_{n=1}^{N} d_{n m} a_{n m}\left(\sum_{k=1}^{K} \gamma_{k n} \varphi_{k}(x)+r_{K n}\right) e_{m} \\
& =\sum_{m=1}^{M} \sum_{k=1}^{K}\left(\sum_{n=1}^{N} d_{n m} a_{n m} \gamma_{k n}\right) e_{k m}+T_{K M N},
\end{aligned}
$$

где

$$
d_{n m}=C_{n} \frac{\sqrt{T} \lambda_{n}}{\sqrt{2} \mu_{n m}}, \quad r_{K n}=\sum_{k=K+1}^{\infty} \gamma_{k n} \varphi_{k}(x), \quad T_{K M N}=\sum_{m=1}^{M} \sum_{n=1}^{N} d_{n m} a_{n m} r_{K n} e_{m} .
$$

Оценим

$$
\left\|r_{K n}\right\|^{2}=\sum_{k=K+1}^{\infty} \gamma_{k n}^{2} \leqslant C_{2}^{2} \sum_{k=K+1}^{\infty} \frac{1}{\left(\lambda_{k}-\lambda_{N}\right)^{2}},
$$

если $K>N$. Таким образом, при $K>N$ имеем

$$
\begin{aligned}
\left\|T_{K M N}\right\| & \leqslant C_{3} \sum_{m=1}^{M} \sum_{n=1}^{N}\left|a_{n m}\right| \cdot\left\|r_{K n}\right\| \leqslant C_{4}\|f\| \sqrt{M N}\left(\sum_{k=K+1}^{\infty} \frac{1}{\left(\lambda_{k}-\lambda_{N}\right)^{2}}\right)^{1 / 2} \\
& \leqslant C_{4}\|f\| \sqrt{M N}\left(\sum_{k=K+1}^{\infty} \frac{1}{(k-N-1 / 2)^{2}}\right)^{1 / 2}
\end{aligned}
$$

Вычислим

$$
\begin{aligned}
v_{M N}-\bar{v}_{M K}= & \sum_{m=1}^{M} \sum_{k=1}^{K}\left(\sum_{\substack{n=1 \\
\mu_{n m} \neq 0}}^{N} d_{n m} a_{n m} \gamma_{k n}\right) e_{k m}+T_{K M N} \\
& -\sum_{m=1}^{M} \sum_{k=1}^{K}\left(\sum_{\substack{n=1 \\
\mu_{n m} \neq 0}}^{\infty} d_{n m} a_{n m} \gamma_{k n}\right) e_{k m} \\
= & -\sum_{m=1}^{M} \sum_{k=1}^{K}\left(\sum_{\substack{n=N+1 \\
\mu_{n m} \neq 0}}^{\infty} d_{n m} a_{n m} \gamma_{k n}\right) e_{k m}+T_{K M N} .
\end{aligned}
$$


Обозначим

$$
D_{K M N}=\sum_{m=1}^{M} \sum_{k=1}^{K}\left(\sum_{\substack{n=N+1 \\ \mu_{n m} \neq 0}}^{\infty} d_{n m} a_{n m} \gamma_{k n}\right) e_{k m}
$$

Тогда

$$
\begin{aligned}
\left\|D_{K M N}\right\|^{2} & =\sum_{m=1}^{M} \sum_{k=1}^{K}\left(\sum_{\substack{n=N+1 \\
\mu_{n m} \neq 0}}^{\infty} d_{n m} a_{n m} \gamma_{k n}\right)^{2} \leqslant \sum_{m=1}^{M} \sum_{k=1}^{K}\left(\sum_{n=N+1}^{\infty} a_{n m}^{2}\right) \cdot \sum_{l=N+1}^{\infty} d_{l m}^{2} \gamma_{k l}^{2} \\
& =\sum_{m=1}^{M}\left(\sum_{n=N+1}^{\infty} a_{n m}^{2}\right) \cdot \sum_{k=1}^{K} \sum_{l=N+1}^{\infty} d_{l m}^{2} \gamma_{k l}^{2} .
\end{aligned}
$$

Из (1.15), (1.16) выводим

$$
\sum_{k=1}^{K} \sum_{l=N+1}^{\infty} d_{l m}^{2} \gamma_{k l}^{2} \leqslant \frac{T \cdot \bar{C}}{2} S_{m} \leqslant \frac{T \cdot \bar{C}}{2}\left(\frac{\pi^{2} C_{2}^{2}}{2 C_{0}^{2}}+L_{0}+4 \pi^{4} C_{2}^{2} b^{2}\right) .
$$

Здесь $\bar{C}=\max _{n} C_{n}$. Следовательно,

$$
\begin{gathered}
\left\|D_{K M N}\right\| \leqslant \sqrt{\frac{T \cdot \bar{C}}{2}\left(\frac{\pi^{2} C_{2}^{2}}{2 C_{0}^{2}}+L_{0}+4 \pi^{4} C_{2}^{2} b^{2}\right)} \cdot\left(\sum_{m=1}^{M} \sum_{n=N+1}^{\infty} a_{n m}^{2}\right)^{1 / 2}, \\
\left\|v_{M N}-v\right\| \leqslant\left\|D_{K M N}\right\|+\left\|T_{K M N}\right\|+\left\|\alpha_{M K}\right\| .
\end{gathered}
$$

Из (1.22) вытекает существование числа $N_{1}=N_{1}(M)$ такого, что $N_{1} \rightarrow \infty$ при $M \rightarrow \infty$ и

$$
\left\|D_{K M N_{1}(M)}\right\|<\frac{1}{3 l} \text {. }
$$

Из (1.21) следует существование числа $K_{2}=K_{2}(M, N)$ такого, что выполнено $K_{2} \geqslant$ $\max \left(N, K_{1}(l)\right)$ и

$$
\left\|T_{K_{2}(M, N) M N}\right\|<\frac{1}{3 l} .
$$

Воспользовавшись (1.19), (1.23)-(1.25), получим оценку

$$
\left\|v_{M_{1}(l) N_{1}\left(M_{1}(l)\right)}-v\right\| \leqslant\left\|D_{K_{2} M_{1}(l) N_{1}}\right\|+\left\|T_{K_{2}\left(M_{1}(l), N_{1}\right) M_{1}(l) N_{1}}\right\|+\left\|\alpha_{M_{1}(l) K_{2}}\right\|<\frac{1}{l} .
$$

Следовательно,

$$
\lim _{l \rightarrow \infty} v_{M_{1}(l) N_{1}\left(M_{1}(l)\right)}=v .
$$

Обозначим

$$
h(x, t)=\sum_{\substack{n, m \in \mathbb{N} \\ \mu_{n m} \neq 0}} \frac{1}{\mu_{n m}} \varphi_{n}(x) a_{n m} \cos \frac{a}{b} m t, \quad g(x, t)=\sum_{\substack{n, m \in \mathbb{N} \\ \mu_{n m} \neq 0}} \frac{1}{\mu_{n m}} \varphi_{n}(x) b_{n m} \sin \frac{a}{b} m t .
$$

Из (1.26) следует, что $h_{x}=v \in L_{2}(\Omega)$. Аналогично доказывается, что $g_{x} \in L_{2}(\Omega)$. Следовательно, $\left(u_{2}\right)_{x}=h_{x}+g_{x} \in L_{2}(\Omega)$ и $u \in H_{1}(\Omega)$. При $k=0$ теорема доказана. 
Пусть $k=1$. Возьмем произвольную функцию $f \in H_{1}(\Omega) \cap R(A)$. Тогда (см. [12])

$$
f_{t}=\sum_{\substack{n, m \in \mathbb{N} \\ \mu_{n m} \neq 0}} \varphi_{n}(x)\left(a_{n m}^{\prime} \cos \frac{a}{b} m t+b_{n m}^{\prime} \sin \frac{a}{b} m t\right),
$$

где $a_{n m}^{\prime}=(a / b) m b_{n m}, b_{n m}^{\prime}=-(a / b) m a_{n m}$. Легко видеть, что

$$
\left(A^{-1} f\right)_{t}=A^{-1} f_{t}=\sum_{\substack{n, m \in \mathbb{N} \\ \mu_{n m} \neq 0}} \frac{1}{\mu_{n m}} \varphi_{n}(x)\left(a_{n m}^{\prime} \cos \frac{a}{b} m t+b_{n m}^{\prime} \sin \frac{a}{b} m t\right) .
$$

Тогда по доказанному выше $\left(A^{-1} f\right)_{t x}=\left(A^{-1} f_{t}\right)_{x} \in L_{2}(\Omega)$. Рассмотрим ряд

$$
\begin{aligned}
& \sum_{\substack{n, m \in \mathbb{N} \\
\mu_{n m} \neq 0}} \frac{m^{2}}{\mu_{n m}} \varphi_{n}(x)\left(a_{n m} \sin \frac{a}{b} m t+b_{n m} \cos \frac{a}{b} m t\right) \\
& \quad=\frac{a}{b} \sum_{\substack{n, m \in \mathbb{N} \\
\mu_{n m} \neq 0}} \frac{m}{\mu_{n m}} \varphi_{n}(x)\left(-b_{n m}^{\prime} \sin \frac{a}{b} m t+a_{n m}^{\prime} \cos \frac{a}{b} m t\right) .
\end{aligned}
$$

Сходимость в $L_{2}(\Omega)$ данного ряда доказывается точно так же, как для ряда (1.9). Следовательно, $\left(A^{-1} f\right)_{t t} \in L_{2}(\Omega)$.

Рассмотрим ряд

$$
\sum_{\substack{n, m \in \mathbb{N} \\ \mu_{n m} \neq 0}} \frac{\lambda_{n}^{2}}{\mu_{n m}} \varphi_{n}(x)\left(a_{n m} \sin \frac{a}{b} m t+b_{n m} \cos \frac{a}{b} m t\right) .
$$

Чтобы доказать сходимость в $L_{2}(\Omega)$ ряда $(1.27)$, рассмотрим числовой ряд

$$
\begin{aligned}
& \sum_{\substack{n, m \in \mathbb{N} \\
\mu_{n m} \neq 0}} \frac{n^{4}}{\mu_{n m}^{2}}\left(a_{n m}^{2}+b_{n m}^{2}\right) \\
& \quad=\sum_{\substack{n \leqslant 3 a / b m \\
\mu_{n m} \neq 0}} \frac{n^{4}}{\mu_{n m}^{2}}\left(a_{n m}^{2}+b_{n m}^{2}\right)+\sum_{n>3(a / b) m} \frac{n^{4}}{\mu_{n m}^{2}}\left(a_{n m}^{2}+b_{n m}^{2}\right) \\
& \leqslant 81 \frac{a^{4}}{b^{4}} \sum_{\substack{n \leqslant 3 a / b m \\
\mu_{n m} \neq 0}} \frac{m^{4}}{\mu_{n m}^{2}}\left(a_{n m}^{2}+b_{n m}^{2}\right)+4 \sum_{n, m \in \mathbb{N}} \frac{n^{2}}{(2 / 3 n-1 / 2)^{2}}\left(a_{n m}^{2}+b_{n m}^{2}\right) \\
& \quad \leqslant 81 \frac{a^{4}}{b^{4}} C_{1}^{2} \sum_{n, m \in \mathbb{N}}\left(\left(b_{n m}^{\prime}\right)^{2}+\left(a_{n m}^{\prime}\right)^{2}\right)+144 \sum_{n, m \in \mathbb{N}}\left(a_{n m}^{2}+b_{n m}^{2}\right)<\infty .
\end{aligned}
$$

Отсюда и из (1.4) вытекает сходимость в $L_{2}(\Omega)$ ряда (1.27). Тогда из $(1.2),(1.3)$ следует $\left(A^{-1} f\right)_{x x} \in L_{2}(\Omega)$. Следовательно, $A^{-1} f \in H_{2}(\Omega)$.

Докажем включение $A^{-1} f \in C^{1}(\Omega)$. Для этого рассмотрим числовой ряд

$$
J=\sum_{\substack{n, m \in \mathbb{N} \\ \mu_{n m} \neq 0}} \frac{n}{\left|\mu_{n m}\right|}\left(\left|a_{n m}\right|+\left|b_{n m}\right|\right)=\frac{b}{a} \sum_{\substack{n, m \in \mathbb{N} \\ \mu_{n m} \neq 0}} \frac{n}{m\left|\mu_{n m}\right|}\left(\left|a_{n m}^{\prime}\right|+\left|b_{n m}^{\prime}\right|\right) .
$$


Используя неравенство Коши-Буняковского, выведем

$$
J \leqslant \frac{b}{a}\left(\sum_{n, m \in \mathbb{N}}\left(a_{n m}^{\prime}\right)^{2}+\left(b_{n m}^{\prime}\right)^{2}\right)^{1 / 2} \cdot\left(\sum_{\mu_{n m} \neq 0} \frac{n^{2}}{m^{2} \mu_{n m}^{2}}\right)^{1 / 2} .
$$

Оценим сумму ряда:

$$
\begin{aligned}
\sum_{\mu_{n m} \neq 0} \frac{n^{2}}{m^{2} \mu_{n m}^{2}} & =\sum_{n \leqslant 3(a / b) m} \frac{n^{2}}{m^{2} \mu_{n m}^{2}}+\sum_{n>3(a / b) m} \frac{n^{2}}{m^{2} \mu_{n m}^{2}} \\
& \leqslant 9 \frac{a^{2}}{b^{2}} \sum_{\mu_{n m} \neq 0} \frac{1}{\mu_{n m}^{2}}+\sum_{n>3(a / b) m} \frac{n^{2}}{m^{2}\left(n-\frac{a}{b} m-\frac{1}{2}+\theta_{n}\right)^{2}\left(n-\frac{1}{2}\right)^{2}} \\
& \leqslant 9 \frac{a^{2}}{b^{2}} \sum_{\mu_{n m} \neq 0} \frac{1}{\mu_{n m}^{2}}+4 \sum_{n, m \in \mathbb{N}} \frac{1}{m^{2}\left(\frac{2}{3} n-\frac{1}{2}\right)^{2}}<.
\end{aligned}
$$

Из сходимости ряда $J,(1.4),(1.7)$ следует, что классическая производная $(\partial / \partial x) u_{2} \in$ $C(\Omega)$. Воспользовавшись неравенством Коши-Буняковского, получим оценку

$$
\sum_{\mu_{n m} \neq 0} \frac{m}{\left|\mu_{n m}\right|}\left(\left|a_{n m}\right|+\left|b_{n m}\right|\right) \leqslant \frac{b}{a}\left(\sum_{n, m \in \mathbb{N}}\left(\left(a_{n m}^{\prime}\right)^{2}+\left(b_{n m}^{\prime}\right)^{2}\right)\right)^{1 / 2} \cdot\left(\sum_{\mu_{n m} \neq 0} \frac{1}{\mu_{n m}^{2}}\right)^{1 / 2}<\infty .
$$

Следовательно, $(\partial / \partial t) u_{2} \in C(\Omega)$. Таким образом, $u_{2} \in C_{1}(\Omega)$.

Для граничных условий (0.3) теорема доказана. Случай граничных условий $(0.4)$ легко свести к задаче с граничными условиями (0.3). Пусть функция $u \in C(\Omega)$ (см. [8]) является обобщенным решением задачи $(1.6),(0.2),(0.4)$. Тогда функция $\bar{u}(x, t)=u(\pi-x, t)$ является решением задачи $(1.6),(0.2),(0.3)$ с правой частью $\bar{f}(x, t)=f(\pi-x, t)$. Следовательно, утверждения теоремы 1 для граничных условий (0.4) являются следствиями доказанных утверждений для граничных условий (0.3). Теорема доказана.

2. Нелинейное уравнение. Рассмотрим квазилинейное волновое уравнение

$$
u_{t t}-u_{x x}=g(u)+f(x, t), \quad 0<x<\pi, \quad t \in \mathbb{R} .
$$

Обозначим $\Lambda=\left\{\mu_{n m} \mid n \in \mathbb{N}, m \in \mathbb{Z}_{+}\right\}$, где $\mathbb{Z}_{+}=\mathbb{N} \cup\{0\}$. Следствием теоремы 1 и теоремы 4.1 работы [8] является следующая теорема.

TeOpema 2. Пусть $g \in C^{1}(\mathbb{R}) u$

$$
\alpha \leqslant \varliminf_{u \rightarrow \infty} \frac{g(u)}{u} \leqslant \varlimsup_{u \rightarrow \infty} \frac{g(u)}{u} \leqslant \beta,
$$

где $[\alpha, \beta] \cap \Lambda=\emptyset$. Тогда при нечетном $b$ для любой $T$-периодической по $t$ функции $f \in$ $C^{1}(\Omega)$ задачи (2.1), (0.2), (0.3) и (2.1), (0.2), (0.4) имеют решение $u \in H_{2}(\Omega) \cap C^{1}(\Omega)$.

ДокАзАТЕЛЬСтво. Существование обобщенного решения $u \in C(\Omega)$ получено в теореме 4.1 работы [8]. Представим это решение в виде $u=u_{1}+u_{2}$, где $u_{1} \in R(A)$, $u_{2} \in N(A)$. Тогда $u_{1}=A^{-1}(g(u)+f)$. Поскольку $g(u)+f \in C(\Omega)$, из теоремы 1 при $k=0$ вытекает включение $u_{1} \in H_{1}(\Omega)$. Отсюда и из конечномерности $N(A)$ следует, что $u \in H_{1}(\Omega) \cap C(\Omega)$. Из этого включения, теоремы Мозера и теоремы 1 получим $u \in H_{2}(\Omega) \cap C^{1}(\Omega)$. Теорема доказана. 
Рассмотрим задачу о свободных колебаниях струны.

$$
u_{t t}-u_{x x}=g(u), \quad 0<x<\pi, \quad t \in \mathbb{R} .
$$

Следствием теоремы 1 и работы [7] являются теоремы 3, 4 .

Теорема 3. Пусть функиия $g \in C^{1}(\mathbb{R})$, удовлетворяет (2.2) и следующим условиям: $g(0)=0$,

$$
\alpha_{1} \leqslant \varliminf_{u \rightarrow 0} \frac{g(u)}{u} \leqslant \varlimsup_{u \rightarrow 0} \frac{g(u)}{u} \leqslant \beta_{1}
$$

где $\alpha_{1}>\beta,[\alpha, \beta] \cap \Lambda=\varnothing,\left[\alpha_{1}, \beta_{1}\right] \cap \Lambda=\varnothing,\left[\beta, \alpha_{1}\right] \cap \Lambda \neq \varnothing$. Пусть также $g(u) / u \leqslant \lambda$ для всех $u \in \mathbb{R} /\{0\}$, где $\lambda \in \Lambda, \lambda>\beta_{1},\left(\beta_{1}, \lambda\right) \cap \Lambda=\varnothing$. Тогда при нечетном $b$ задачи $(2.3),(0.2),(0.3)$ и $(2.3),(0.2),(0.4)$ имеют нетривиальные (не равные тожсдественно нулю) решения $u \in H_{2}(\Omega) \cap C^{1}(\Omega)$.

ТЕОрема 4. Пусть функиия $g \in C^{1}(\mathbb{R}), g(0)=0$ и выполнены условия $(2.2),(2.4)$, где $\alpha>\beta_{1},[\alpha, \beta] \cap \Lambda=\varnothing,\left[\alpha_{1}, \beta_{1}\right] \cap \Lambda=\varnothing,\left[\beta_{1}, \alpha\right] \cap \Lambda \neq \varnothing$. Пусть также $g(u) / u \geqslant \lambda$ для всех $u \in \mathbb{R} /\{0\}$, где $\lambda \in \Lambda, \lambda<\alpha_{1},\left(\lambda, \alpha_{1}\right) \cap \Lambda=\varnothing$. Тогда при нечетном $b$ задачи $(2.3),(0.2),(0.3)$ и $(2.3),(0.2),(0.4)$ имеют нетривиальные решения $u \in H_{2}(\Omega) \cap$ $C^{1}(\Omega)$.

В качестве приложения теоремы 4 рассмотрим уравнение sin-Гордон (см. [13]):

$$
u_{t t}-u_{x x}+\sin u=0, \quad 0<x<\pi, \quad t \in \mathbb{R} .
$$

Заметим, что из работы [16] следует существование нетривиального, гладкого $T=$ $5 \pi / 3$-периодического по времени решения уравнения (2.5) с граничными условиями Дирихле $u(0, t)=u(\pi, t)=0$, которое зависит от времени.

Будем искать $2 \pi$-периодическое решение, удовлетворяющее граничным условиям (0.3) или (0.4). В этом случае

$$
\Lambda=\left\{\left(n-\frac{1}{2}\right)^{2}-m^{2}+\alpha_{n} \mid n \in \mathbb{N}, m \in \mathbb{Z}_{+}\right\}, \quad \text { где } \quad \alpha_{n}=(2 n-1) \theta_{n}+\theta_{n}^{2} .
$$

Tеорема 5. Если $T=2 \pi u$

$$
-1 \notin \Lambda
$$

то задачи $(2.5),(0.2),(0.3)$ и (2.5),(0.2),(0.4) имеют нетривиальное решение $и \in$ $H_{2}(\Omega) \cap C^{1}(\Omega)$, которое зависит от времени.

Доказательство. Заметим вначале, что если $T=2 \pi$, то $\operatorname{ker} A=\{0\}$. Действительно, равенство $\mu_{n m}=0$ равносильно уравнению $2 \theta_{n}=2 m-2 n+1$, которое не имеет решения, поскольку $2 \theta_{n}<1$ (см. [7]). Обозначим $z_{n m}=(n-1 / 2)^{2}-m^{2}$. Тогда

$$
\left\{z_{n m} \mid n \in \mathbb{N}, m \in \mathbb{Z}_{+}\right\}=\left\{l+\frac{1}{4} \mid l \in \mathbb{Z}\right\} .
$$

Используя введенные обозначения, запишем соотношение

$$
\mu_{n m}=z_{n m}+\alpha_{n}
$$

Поскольку $\alpha_{1}=\theta_{1}+\theta_{1}^{2}<3 / 4$, из (2.7) следует, что $\mu_{11} \in(-3 / 4,0)$. Отсюда и из $(2.6),(2.7)$ вытекает выполнение условий теоремы 4 . Из теоремы 4 следует существование нетривиального решения $u \in H_{2}(\Omega) \cap C^{1}(\Omega)$ задач $(2.5),(0.2),(0.3)$ и $(2.5),(0.2),(0.4)$. Докажем, что это решение зависит от времени. 
Предположим противное, т.е. функция $u=u(x) \in H_{2}(0, \pi) \cap C^{1}[0, \pi]$ является нетривиальным решением задачи

$$
\begin{gathered}
-u_{x x}+\sin u=0, \quad 0<x<\pi, \\
u(0)=u(\pi)+h u^{\prime}(\pi)=0 .
\end{gathered}
$$

Умножив равенство (2.8) на $u_{x}$, выводим

$$
u_{x}^{2}=2(C-\cos u) .
$$

Если $C<1$, то подставив $x=0$ в равенство (2.10), придем к противоречию: $u_{x}^{2}(0)=$ $2(C-1)<0$. При $C=1$ уравнение $(2.10)$ примет вид $u_{x}= \pm 2 \sin (u / 2)$. Рассмотрим случай

$$
u_{x}=2 \sin \frac{u}{2} .
$$

Проинтегрировав уравнение (2.11), получим частное решение $u \equiv 0$ и нетривиальное решение $\operatorname{tg}(u / 4)=C e^{2 x}, C \neq 0$. Подставив сюда $x=0$, с учетом (2.9) придем к противоречию. Уравнение $u_{x}=-2 \sin (u / 2)$ рассматривается аналогично.

Если $C>1$, то из (2.10) следует, что $u_{x} \neq 0$. Если $u_{x}>0$ при всех $x$, то функция $u(x)$ возрастает и из $(2.9)$ вытекает, что $u(\pi)>0$. Поэтому $u(\pi)+h u^{\prime}(\pi)>0$, что противоречит (2.9). Если $u_{x}<0$ при всех $x$, то аналогично доказывается, что $u(\pi)+h u^{\prime}(\pi)<0$, что также противоречит (2.9). Таким образом, задача $(2.8),(2.9)$ имеет только тривиальное решение в классе $C^{1}[0, \pi]$.

В заключение заметим, что если функция $u=u(x) \in H_{2}(0, \pi) \cap C^{1}[0, \pi]$ является решением уравнения (2.8) с граничными условиями

$$
u(0)-h u^{\prime}(0)=u(\pi)=0,
$$

то функция $\bar{u}=u(\pi-x)$ является решением задачи $(2.8),(2.9)$. Поэтому задача $(2.8),(2.12)$ имеет также только тривиальное решение. Теорема доказана.

ЗАмЕчАниЕ. Условие (2.6) выполнено, например, при

$$
h>\frac{8}{\pi} .
$$

Действительно, согласно [7] имеем

$$
\alpha_{n} \leqslant(2 n-1) \theta_{n}+\frac{1}{4} \leqslant\left(2-\frac{1}{n}\right) \frac{2}{\pi h}+\frac{1}{4} .
$$

Следовательно, $0<\alpha_{n}<4 /(\pi h)+1 / 4$. Отсюда и из $(2.7),(2.13)$ следует $0<\alpha_{n}<3 / 4$, $\mu_{n m} \neq-1$.

Условие (2.6) выполнено также, если

$$
h>\frac{8}{3 \pi}, \quad \theta_{2} \neq \sqrt{3}-1,5 .
$$

Действительно, в этом случае $\alpha_{n}<1,75$ и, следовательно, $\mu_{n m} \neq-1$ при $n>2$. При $n=2$ имеем $\mu_{22}=-1,75+3 \theta_{2}+\theta_{2}^{2} \neq-1$, если выполнено (2.14). Для остальных значений $n, m$ также, очевидно, выполнено условие $\mu_{n m} \neq-1$. 
3. Граничные условия 3-го рода с отрицательным $h$. Рассмотрим задачу Штурма-Лиувилля

$$
\varphi^{\prime \prime}+\lambda^{2} \varphi=0, \quad \varphi(0)=\varphi(\pi)+h \varphi^{\prime}(\pi)=0 .
$$

Пусть $h \in(-\infty,-\pi)$. Предположим $\lambda=-\mu^{2}<0$, где $\mu>0$. Тогда $\varphi=C_{1} e^{\mu x}+$ $\mathrm{C}_{2} e^{-\mu x}$. Учитывая граничные условия, получим

$$
\varphi=e^{\mu x}-e^{-\mu x}
$$

где $\mu$ удовлетворяет уравнению

$$
\frac{2 k}{e^{2 \mu \pi}+1}+\mu-k=0,
$$

а $k=-1 / h<1 / \pi$. Обозначим $f(\mu)=2 k /\left(e^{2 \mu \pi}+1\right)+\mu-k$. Вычислим производную $f^{\prime}(\mu)=\left(e^{4 \mu \pi}-2(2 k \pi-1) e^{2 \mu \pi}+1\right)^{2} /\left(e^{2 \mu \pi}+1\right)$. Рассмотрим квадратный трехчлен $g(t)=t^{2}-2(2 k \pi-1) t+1$. При $h<-\pi$ его дискриминант $D_{1}=4 k \pi(k \pi-1)<0$. Поэтому $f^{\prime}(\mu)>0$ при всех $\mu$. А поскольку $f(0)=0$, при $\mu>0$ уравнение $(3.2)$ решений не имеет. При $\lambda=0$ уравнение имеет решение $\varphi=C_{1} x+C_{2}$ и при $h<-\pi$ граничные условия не выполняются.

Таким образом, при $h<-\pi$ задача Штурма-Лиувилля (3.1) может иметь только положительные собственные значения $\lambda_{n}=\mu_{n}^{2}$. Легко видеть, что соответствующие собственные функции имеют вид

$$
\varphi_{n}(x)=\sin \mu_{n} x,
$$

где $\mu_{n}>0$ и являются корнями уравнения

$$
\operatorname{tg} \mu \pi=-h \mu .
$$

Представим числа $\mu_{n}$ в виде

$$
\mu_{n}=n-\frac{1}{2}-\theta_{n}, \quad n \in \mathbb{N} .
$$

Легко видеть, что $\theta_{n} \in(0,1 / 2)$. Подставив выражение (3.6) в (3.5), получим соотношение

$$
\theta_{n}=\frac{1}{n} \cdot \frac{k}{\pi} \cdot \frac{\theta_{n} \pi}{\operatorname{tg} \theta_{n} \pi} \cdot \frac{1}{1-\frac{1}{2 n}-\frac{\theta_{n}}{n}} .
$$

Поскольку функция $t / \operatorname{tg} t$ при $t \in(0, \pi / 2)$ убывает и принимает значения из интервала $(0,1)$, из $(3.7)$ получим оценку

$$
0<b_{0} \frac{1}{n}<\theta_{n}<b_{1} \frac{1}{n}
$$

для всех $n \in \mathbb{N}$, где $b_{0}=\theta_{1} /\left(\pi \operatorname{tg} \theta_{1} \pi\right), b_{1}=2 /\left(\pi^{2}\left(1-2 \theta_{1}\right)\right)$.

При $h=-\pi$ есть нулевое собственное значение $\lambda_{1}=0$, соответствующее собственной функции $\varphi_{1}(x)=-x$. Если $\lambda=-\mu^{2}<0$, то собственная функция должна иметь вид (3.2), где $\mu$ удовлетворяет уравнению (3.3). При $h=-\pi$ производная $f^{\prime}(\mu)=\left(e^{2 \mu \pi}-1\right)^{2} /\left(e^{2 \mu \pi}+1\right)^{2}>0$, если $\mu>0$. А так как $f(0)=0$, отрицательных собственных значений нет. 
Как в предыдущем случае собственные функции с положительными собственными значениями имеют вид (3.4), где $\mu_{n}$ выражается формулой $(3.6), \theta_{n}$ удовлетворяет оценке (3.8), в которой $b_{0}=\theta_{1} k /\left(\operatorname{tg} \theta_{1} \pi\right), b_{1}=2 k /\left(\pi\left(1-2 \theta_{1}\right)\right)$.

Докажем, что при $h \in(-\pi, 0)$ имеется ровно одно отрицательное собственное значение, а остальные собственные значения положительные. В этом случае, очевидно, нуль не является собственным значением.

Рассмотрим случай $\lambda=-\mu^{2}$. Соответствующая собственная функция выражается формулой (3.2), а $\mu$ удовлетворяет равенству (3.3). Поскольку $h \in(-\pi, 0)$, то $D_{1}>0$. Поэтому квадратный трехчлен $g$ имеет различные корни $t_{1}, t_{2}$. Из теоремы Виета делаем вывод $0<t_{1}<1<t_{2}$. Обозначим $\mu_{0}=1 /(2 \pi) \ln t_{2}>0$. Тогда $f^{\prime}(\mu)=\left(e^{2 \mu \pi}-1\right)\left(e^{2 \mu \pi}-e^{2 \mu_{0} \pi}\right) /\left(e^{2 \mu \pi}+1\right)^{2}>0$, если $\mu>0$. Таким образом, $f^{\prime}(\mu)<0$ при $\mu \in\left(0, \mu_{0}\right), f^{\prime}(\mu)>0$ при $\mu>\mu_{0}$. А поскольку $f(0)=0, f(k)>0$, при $\mu>0$ уравнение (3.3) имеет единственный корень $\mu_{*} \in\left(\mu_{0}, k\right)$. Следовательно, единственное отрицательное собственное значение $\lambda_{1}=-\mu_{*}^{2}$, а соответствующая ему собственная функция $\varphi_{1}=e^{\mu_{*} x}-e^{-\mu_{*} x}$.

4. Собственные значения волнового оператора. Собственные функции оператора Даламбера $\partial_{t t}-\partial_{x x}$, действующего на бесконечно гладких функциях, удовлетворяющих условиям $(0.2),(0.3)$, имеют вид

$$
\mu_{n m}=\lambda_{n}-\left(\frac{a}{b} m\right)^{2}
$$

где $n \in \mathbb{N}, m \in \mathbb{Z}_{+}$. Соответствующие им собственные функции выражаются формулами

$e_{n m}^{c}=C_{n m} \varphi_{n}(x) \cos \frac{a}{b} m t, \quad e_{n m}^{s}=C_{n m} \varphi_{n}(x) \sin \frac{a}{b} m t, \quad e_{n 0}=C_{n 0} \varphi_{n}(x), \quad n, m \in \mathbb{N}$.

Нормирующие множители $C_{n m}$ подобраны так, что система функций $e_{n m}^{c}, e_{n m}^{s}, e_{n 0}$ является ортонормированной в $L_{2}(\Omega)$.

ЗАмечАниЕ. Очевидно, что собственные значения задачи Штурма-Лиувилля (1.2) совпадают с собственными значениями задачи (3.1), а соответствующие им собственные функции равны $\varphi_{n}(\pi-x)$.

Рассмотрим самосопряженное расширение $A$ оператора Даламбера такое, что $A=$ $A_{0}^{*}$, где

$$
D\left(A_{0}\right)=\left\{\sum_{n=1}^{N}\left(c_{n} e_{n 0}+\sum_{m=1}^{M}\left(a_{n m} e_{n m}^{c}+b_{n m} e_{n m}^{s}\right)\right) \mid N, M \in \mathbb{N}, c_{n}, a_{n m}, b_{n m} \in \mathbb{R}\right\}
$$

и $A_{0} u=u_{t t}-u_{x x}$ для всех $u \in D\left(A_{0}\right)$.

Заметим, что при $h \in[-\pi, 0)$ и $n=1$ уравнение $\mu_{n m}=0$ не имеет решения. В остальных случаях это уравнение равносильно соотношению $2 b n-2 a m-b=2 b \theta_{n}$. Из (3.8) следует, что данное уравнение имеет конечное число пар решений $(n, m)$. Поэтому $\operatorname{dim} \operatorname{ker} A<\infty$. При $b=1$, очевидно, $\operatorname{ker} A=\{0\}$. Из (3.6), (4.1) выразим

$$
\mu_{n m}=\left(n-\frac{1}{2}\right)-\left(\frac{a}{b} m\right)^{2}-\alpha_{n}
$$

где $\alpha_{n}=(2 n-1) \theta_{n}-\theta_{n}^{2}$. Из неравенств (3.8) вытекает включение

$$
\alpha_{n} \in\left(c_{0}, 2 b_{1}\right),
$$

где $c_{0}=\min \left(b_{0},(1 / 2) \theta_{1}\right)$. Заметим, что из (3.7) следует $\lim _{n \rightarrow \infty} \alpha_{n}=-2 /(h \pi)$. 
Рассмотрим уравнение

$$
\left(n-\frac{1}{2}\right)-\left(\frac{a}{b} m\right)^{2}=0
$$

относительно $n \in \mathbb{N}, m \in Z_{+}$. При нечетных $b$ оно не выполняется ни при каких целых числах $n, m$. Если $b$ четное число, то уравнение (4.4) имеет бесконечно много решений $n=a l-(a-1) / 2, m=b / 2(2 l-1), l \in \mathbb{N}$.

При $h \in(-\pi, 0)$ для любой функции $f \in R(A)$

$$
A^{-1} f=u_{1}+u_{2}+u_{3},
$$

где

$$
\begin{aligned}
& u_{1}=\sum_{n=2}^{\infty} \frac{1}{\mu_{n}^{2}} f_{n} \varphi_{n}(x)-\frac{1}{\mu_{*}^{2}} f_{1} \varphi_{1}(x), \\
& u_{2}=-\varphi_{1}(x) \sum_{m=1}^{\infty} \frac{1}{\mu_{*}^{2}+\left(a^{2} / b^{2}\right) m^{2}}\left(a_{1 m} \cos \frac{a}{b} m t+b_{1 m} \sin \frac{a}{b} m t\right), \\
& u_{3}=\sum_{n=2}^{\infty} \sum_{m=1}^{\infty} \frac{1}{\mu_{n m}} \varphi_{n}(x)\left(a_{n m} \cos \frac{a}{b} m t+b_{n m} \sin \frac{a}{b} m t\right),
\end{aligned}
$$

$\left\{f_{n}, a_{n m}, b_{n m}\right\}_{n, m \in \mathbb{N}}-$ коэффициенты Фурье функции $f$. Легко видеть, что $u_{1}, u_{2} \in$ $H_{2}(\Omega) \cap C^{1}(\Omega)$. Поэтому при $h \in(-\pi, 0)$ справедливо утверждение теоремы 1 .

При $h=-\pi$ для функции $f \in R(A)$ прообраз $A^{-1} f$ выражается формулой $(4.5)$, в которой

$$
u_{1}=\sum_{n=2}^{\infty} \frac{1}{\mu_{n}^{2}} f_{n} \varphi_{n}(x), \quad u_{2}=-\frac{b^{2}}{a^{2}} x \sum_{m=1}^{\infty} \frac{1}{m^{2}}\left(a_{1 m} \cos \frac{a}{b} m t+b_{1 m} \sin \frac{a}{b} m t\right),
$$

$u_{3}$ выражается формулой (4.6).

При $h<-\pi$ для любой функции $f \in R(A)$ имеем

$$
A^{-1} f=\sum_{n=1}^{\infty} \frac{1}{\mu_{n}^{2}} f_{n} \varphi_{n}(x)+\sum_{\mu_{n m} \neq 0} \frac{1}{\mu_{n m}} \varphi_{n}(x)\left(a_{n m} \cos \frac{a}{b} m t+b_{n m} \sin \frac{a}{b} m t\right) .
$$

Следовательно, при $h \leqslant-\pi$ также справедливо утверждение теоремы 1.

При $h<0$ и нечетном $b$ стандартно доказывается, что $\sum_{\mu \neq 0} 1 /\left(\mu_{n m}^{2}\right)<\infty$. Поэтому оператор $A^{-1}: R(A) \rightarrow R(A)$ является вполне непрерывным. Более того, точно так же, как лемма 2.5 в [7] доказывается вполне непрерывность оператора $A^{-1}: R(A) \rightarrow C(\Omega)$.

5. Квазилинейное уравнение. Как и выше обозначим $\Lambda=\left\{\mu_{n m} \mid n \in \mathbb{N}, m \in\right.$ $\left.\mathbb{Z}_{+}\right\}$. Следствием теоремы 1 и теоремы 2 из [14] является следующая теорема.

Теорема 6. Пусть бункиия $g(u) \in C^{1}(\mathbb{R})$ и удовлетворяет условию $(2.2)$, где $[\alpha, \beta] \cap \Lambda=\varnothing$. Тогда при нечетном $b$ для любой $T$-периодической по $t$ функиии $f \in C^{1}(\Omega)$ задачи (0.1)-(0.3) и (0.1), (0.2), (0.4) имеют решение $u \in H_{2}(\Omega) \cap C^{1}(\Omega)$.

Обозначим $\Lambda_{-}=\left\{-\mu_{n m} \mid n \in \mathbb{N}, m \in \mathbb{Z}_{+}\right\}$и рассмотрим случай, когда $b$ является четным числом. Опираясь на (4.3), не трудно проверить, что в этом случае для оператора $(-A)$ выполнено свойство $I I$ из работы [11], в котором

$$
\Lambda_{1}=\left\{e_{n m}^{c}, e_{n m}^{s} \mid n=a l-\frac{a-1}{2}, m=\frac{b}{2}(2 l-1), l \in \mathbb{N}\right\} .
$$


Пусть функция $g(u)$ непрерывна и удовлетворяет условию (2.2).

ОПРЕДЕЛЕНИЕ. Обобщенным решением задач $(0.1)-(0.3)$ и $(0.1),(0.2),(0.4)$ называется $T$-периодическая по $t$ функция $u \in L_{2}(\Omega)$ такая, что $\left(u, A_{0} \varphi\right)=(g(u)+f, \varphi)$ для всех $\varphi \in D\left(A_{0}\right)$.

Следствием теоремы 3.2 из [11] является следующая теорема.

Теорема 7. Пусть ь есть четное число, функиия $g(u)$ непрерывна и удовлетворяет условию (2.2), где $[\alpha, \beta] \cap \Lambda_{-}=\varnothing$. Если функиия $g(u)+c_{0}$ и не убывает, то для любой T-периодической по $t$ функиии $f \in L_{2}(\Omega)$ задачи (0.1)-(0.3) u (0.1), (0.2),(0.4) имеют обобщенное решение $u \in L_{2}(\Omega)$.

6. Волновое уравнение с переменными коэффициентами. Рассмотрим квазилинейное уравнение колебаний неоднородной струны

$$
p(x) u_{t t}-\left(p(x) u_{x}\right)_{x}=g(x, t, u)+f(x, t), \quad 0<x<\pi, \quad t \in \mathbb{R},
$$

с граничными условиями $(0.3)$ или $(0.4)$, в которых $h>0$. T-периодическое решение $(T=2 \pi a / b, a, b \in \mathbb{N},(a, b)=1)$ задач $(6.1),(0.2),(0.3)$ или $(6.1),(0.2),(0.4)$ будем искать в виде ряда Фурье по системе

$$
S=\left\{\varphi_{n}(x), \varphi_{n}(x) \cos \frac{a}{b} m t, \varphi_{n}(x) \sin \frac{a}{b} m t\right\}_{n, m \in \mathbb{N}},
$$

где функции $\varphi_{n}(x)$ являются решением одной из задач Штурма-Лиувилля

$$
\begin{array}{ll}
-\left(p(x) \varphi^{\prime}(x)\right)^{\prime}=p(x) \lambda \varphi(x), & \varphi(0)=\varphi(\pi)+h \varphi^{\prime}(\pi)=0, \\
-\left(p(x) \varphi^{\prime}(x)\right)^{\prime}=p(x) \lambda \varphi(x), & \varphi(0)-h \varphi^{\prime}(0)=\varphi(\pi)=0 .
\end{array}
$$

Функция $p(x)$ удовлетворяет следующим условиям (см. [15]):

$$
\begin{gathered}
p \in C^{3}[0, \pi], \quad p(x) \geqslant d>0 \quad \text { для любого } x \in[0, \pi], \\
\min _{[0, \pi]} \eta_{p}(x)>0,
\end{gathered}
$$

где $\eta_{p}(x)=(1 / 2)\left(p^{\prime \prime} / p\right)-(1 / 4)\left(p^{\prime} / p\right)^{2}$. В работе [11] на функцию $p(x)$ накладывается еще одно условие в граничной точке отрезка $[0, \pi]$. Если исследуется граничное условие (0.4), то требуется, чтобы

$$
\frac{p^{\prime}(0)}{p(0)}>-\frac{2}{h}
$$

А если рассматривается граничное условие (0.3), то требуется, чтобы

$$
\frac{p^{\prime}(\pi)}{p(\pi)}<\frac{2}{h} .
$$

Рассуждения, проведенные нами в пункте 4, позволяют рассмотреть случаи, когда в условиях (6.5)-(6.7) стоят противоположные неравенства.

Замена неизвестной функции

$$
z(x)=\sqrt{p(x)} \varphi(x)
$$


приводит задачи Штурма-Лиувилля (6.2), (6.3) соответственно к следующим задачам:

$$
\begin{aligned}
z^{\prime \prime}+\left(\lambda-\eta_{p}(x)\right) z=0, & z(0)=z(\pi)+h_{1} z^{\prime}(\pi)=0, \\
z^{\prime \prime}+\left(\lambda-\eta_{p}(x)\right) z=0, & z(0)-h_{2} z^{\prime}(0)=z(\pi)=0 .
\end{aligned}
$$

Здесь

$$
h_{1}=\frac{2 p(\pi) h}{2 p(\pi)-h p^{\prime}(\pi)}, \quad h_{2}=\frac{2 p(0) h}{2 p(0)+h p^{\prime}(0)} .
$$

Рассмотрим задачу (6.2) с функцией $p(x)$, удовлетворяющей условию (6.4). Простые рассуждения показывают, что при

$$
\frac{p^{\prime}(\pi)}{p(\pi)}=\frac{2}{h}
$$

замена переменной (6.8) приводит к задаче

$$
z^{\prime \prime}+\left(\lambda-\eta_{p}(x)\right) z=0, \quad z(0)=z^{\prime}(\pi)=0 ;
$$

при $p^{\prime}(\pi) / p(\pi) \in(2 / h, 2(1 / h+1 / \pi))$ выполнено неравенство $h_{1}<-\pi ;$ при $p^{\prime}(\pi) / p(\pi)=$ $2(1 / h+1 / \pi)$ получим равенство $h_{1}=-\pi$; а при $p^{\prime}(\pi) / p(\pi)>2(1 / h+1 / \pi)$ имеем включение $h_{1} \in(-\pi, 0)$.

Обозначим $\eta_{1}=\min _{[0, \pi]} \eta_{p}(x), \eta_{2}=\max _{[0, \pi]} \eta_{p}(x)$. Пусть $\lambda_{n}$ есть последовательность собственных значений задачи (6.2). Точно также, как в [11], с использованием теоремы Штурма о сравнении доказывается, что если $p(x)$ удовлетворяет условию $(6.9)$ и $\eta_{1}>0$, то $\lambda_{n}=\mu_{n}^{2}$, где $\mu_{n}=n-1 / 2+\theta_{n}$ и

$$
\frac{\eta_{1}}{\sqrt{1+\eta_{1}}+1} \frac{1}{n} \leqslant \theta_{n} \leqslant \frac{2 \eta_{2}}{3} \frac{1}{n} \quad \text { для всех } n \in \mathbb{N} \text {; }
$$

если

$$
\begin{aligned}
\frac{p^{\prime}(\pi)}{p(\pi)}>\frac{2}{h}, \\
\eta_{2}<0,
\end{aligned}
$$

то существует $n_{0} \in \mathbb{N}$ и положительные константы $b_{0}, b_{1} \in \mathbb{R}$ такие, что

$$
\lambda_{n}=\mu_{n}^{2}, \quad \mu_{n}=n-\frac{1}{2}-\theta_{n}>0 \quad \text { для всех } n \geqslant n_{0}
$$

и при $n \geqslant n_{0}$ справедлива оценка (3.8).

Рассмотрим пространство $L_{2}(\Omega)$ со скалярным произведением

$$
(f, g)=\int_{\Omega} p(x) f(x, t) g(x, t) d x d t, \quad f, g \in L_{2}(\Omega) .
$$

Функции системы $S$ являются ортогональными в $L_{2}(\Omega)$. Определим оператор $A_{0}$ : $L_{2}(\Omega) \rightarrow L_{2}(\Omega)$, для которого

$$
D\left(A_{0}\right)=\left\{u=\sum_{n=1}^{N} \sum_{m=1}^{M} \varphi_{n}\left(a_{n m} \cos \frac{a}{b} m t+b_{n m} \sin \frac{a}{b} m t\right) \mid M, N \in \mathbb{N}\right\}
$$


и $A_{0} \varphi=p \varphi_{t t}-\left(p \varphi_{x}\right)_{x}$ для всех $\varphi \in D\left(A_{0}\right)$. Пусть $\bar{A}_{0} \varphi=(1 / p) A_{0} \varphi$ для любого $\varphi \in D\left(A_{0}\right)$. Обозначим $A=\left(\bar{A}_{0}\right)^{*}$ в $L_{2}(\Omega)$. Система функций $S$ является системой собственных функций операторов $\bar{A}_{0}$ и $A$ с собственными значениями $\mu_{n m}$ (см. [10]).

Пусть функция $g(x, t, u) \in C([0, \pi] \times \mathbb{R} \times \mathbb{R})$ и удовлетворяет неравенству

$$
\alpha \leqslant \varliminf_{u \rightarrow \infty} \frac{g(x, t, u)}{p(x) u} \leqslant \varlimsup_{u \rightarrow \infty} \frac{g(x, t, u)}{p(x) u} \leqslant \beta
$$

равномерно по $(x, t) \in \Omega$.

ОПРЕДЕЛЕНИЕ. Обобщенным решением задач (6.1), (0.2), (0.3) и (6.1), (0.2), (0.4) называется $T$-периодическая по $t$ функция $u \in C([0, \pi] \times \mathbb{R})$ такая, что

$$
\int_{\Omega} u A_{0} \varphi d x d t=\int_{\Omega}(g(x, t, u)+f) \varphi d x d t \quad \text { для всех } \quad \varphi \in D\left(A_{0}\right) .
$$

Следствием теоремы 2 работы [14] является теорема 8.

ТЕОРема 8. Пусть выполнены условия (6.4), функиия $g(x, t, u) \in C([0, \pi] \times \mathbb{R} \times \mathbb{R})$ $T$-периодична по $t$ и удовлетворяет $(6.13)$, где $[\alpha, \beta] \cap \Lambda=\varnothing$. Предположим также, что либо верно равенство (6.9) и $\eta_{1}>0$, либо имеют место неравенства (6.10), (6.11). Тогда при нечетном $b$ для любой $T$-периодической по $t$ функиии $f \in L_{2}(\Omega)$ задача (6.1),(0.2),(0.3) имеет обобщенное решение $u \in C(\Omega)$.

\section{СПИСОК ЦИТИРОВАННОЙ ЛИТЕРАТУРЫ}

[1] H. Brézis, L. Nirenberg, "Forced vibration for a nonlinear wave equation", Comm. Pure Appl. Math., 31:1 (1978), 1-30.

[2] P.H. Rabinowitz, "Free vibration for a semilinear wave equation", Comm. Pure Appl. Math., 31:1 (1978), 31-68.

[3] П. И. Плотников, "Существование счетного множества периодических решений задачи о вынужденных колебаниях для слабо нелинейного волнового уравнения", Maтем. сб., 136:4 (1988), 546-560.

[4] E. Feireisl, "On the existence of periodic solutions of a semilinear wave equation with a superlinear forcing term", Czechoslovak Math. J., 38:1 (1988), 78-87.

[5] И. А. Рудаков, "Нелинейные колебания струны”, Вестн. Моск. ун-та. Сер. 1. Матем., мех., 1984, № 2, 9-13.

[6] И. А. Рудаков, "Периодическое по времени решение уравнения вынужденных колебаний струны с однородными граничными условиями”, Дифферени. уравнения, 39:11 (2003), 1550-1555.

[7] И. А. Рудаков, "Нетривиальное периодическое решение нелинейного волнового уравнения с однородными граничными условиями", Дифферени. уравнения, 41:10 (2005), $1392-1399$.

[8] И. А. Рудаков, "Периодические решения нелинейного волнового уравнения с однородными граничными условиями", Изв. РАН. Сер. матем., 70:1 (2006), 117-128.

[9] И. А. Рудаков, "Периодические решения квазилинейного волнового уравнения с однородными граничными условиями", Фундамент. и прикл. матем., 12:5 (2006), 189-201.

[10] И. А. Рудаков, "Периодические решения нелинейного волнового уравнения с непостоянными коэффициентами", Матем. заметки, 76:3 (2004), 427-438.

[11] И. А. Рудаков, "Периодические решения квазилинейного волнового уравнения с переменными коэффициентами", Матем. сб., 198:7 (2007), 91-108. 
[12] M. Berti, P. Bolle, "Multiplicity of periodic solutions of nonlinear wave equations", Nonlinear Anal., 56:7 (2004), 1011-1046.

[13] L. Nirenberg, "Variational and topological methods in nonlinear problems", Bull. Amer. Math. Soc. (N.S.), 4:3 (1981), 267-302.

[14] И. А. Рудаков, "Периодическое по времени решение нелинейного волнового уравнения с граничными условиями Неймана и Дирихле", Изв. вузов. Матем., 2007, № 2, 46-55.

[15] V. Barbu, N.H. Pavel, "Periodic solutions to nonlinear one-dimensional wave equation with $x$-dependent coefficients", Trans. Amer. Math. Soc., 349:5 (1997), 2035-2048.

[16] И. А. Рудаков, "Задача о свободных периодических колебаниях струны с немонотонной нелинейностью", УМН, 40:1 (1985), 215-216.

\section{В. А. Кондратьев}

Московский государственный университет

им. М. В. Ломоносова

\section{И. А. Рудаков}

Брянский государственный университет им. академика

И. Г. Петровского

E-mail: rudakov_bgu@mail.ru
Поступило

26.11.2007

Исправленный вариант

17.03.2008 